\title{
BMJ Open Development and evaluation of guidelines for prevention of retraumatisation in torture survivors during surgical care: protocol for a multistage qualitative study
}

\author{
Ana Carla Schippert (D) , ${ }^{1,2}$ Ellen Karine Grov, ${ }^{1}$ Tone Dahl-Michelsen, ${ }^{3}$ \\ Juha Silvola, ${ }^{2,4}$ Bente Sparboe-Nilsen (D) , ${ }^{1}$ Stein Ove Danielsen, ${ }^{1}$ Mariann Aaland, ${ }^{2}$ \\ Ann Kristin Bjørnnes (1) ${ }^{1}$
}

To cite: Schippert AC, Grov EK, Dahl-Michelsen T, et al. Development and evaluation of guidelines for prevention of retraumatisation in torture survivors during surgical care: protocol for a multistage qualitative study. BMJ Open 2021;11:e053670. doi:10.1136/ bmjopen-2021-053670

- Prepublication history and additional supplemental material for this paper are available online. To view these files, please visit the journal online (http://dx.doi.org/10.1136/ bmjopen-2021-053670).

Received 25 May 2021 Accepted 04 October 2021

D) Check for updates

(c) Author(s) (or their employer(s)) 2021. Re-use permitted under CC BY-NC. No commercial re-use. See rights and permissions. Published by BMJ.

${ }^{1}$ Institute of Nursing and Health Promotion, Oslo Metropolitan University, Oslo, Norway

${ }^{2}$ Akershus University Hospital, Lørenskog, Norway

${ }^{3}$ Institute of Physiotherapy, Oslo Metropolitan University, Oslo, Norway

${ }^{4}$ Institute of Clinical Medicine, Campus Ahus, University of Oslo, Oslo, Norway

Correspondence to Dr Ana Carla Schippert; ana.schippert@gmail.com

\section{ABSTRACT}

Introduction Healthcare professionals working in somatic departments are not trained to recognise signs of torture or provide appropriate healthcare to torture survivors, which may result in retraumatisation during surgical treatment.

Methods and analysis This protocol outlines a four-stage qualitative-method strategy for the development and evaluation of guidelines for prevention of retraumatisation of torture survivors during surgical care. The systematic search for literature review in stages 1 and 2 was conducted in August 2019 and March 2021, respectively, using nine databases. The search strategies employed in stage 1, without imposing any date limits, resulted in the inclusion of eight studies that addressed inadequate healthcare strategies associated with retraumatisation. The clinical guidelines review in stage 2 will include publications from 2000 onwards, which will be appraised using the Appraisal of Guidelines Research and Evaluation Version II instrument. Following multi-institutional recruitment in Norway, stage 3 will explore survivors' experiences of receiving surgical treatment using indepth interviews $(n=8-12)$, which will be audio-recorded, transcribed verbatim and analysed using the interpretative phenomenological analysis approach. In stage $4 a$, based on the findings from stages 1, 2 and 3, a set of clinical guidelines for preventing retraumatisation during surgical treatment will be developed. Next, the feasibility and acceptability of the guidelines will be assessed in stage $4 b$ in three interdisciplinary focus group interviews ( $n=5$ per group) and text condensation analyses.

Ethics and dissemination The Regional (South-East C) Committee for Medical and Health Research Ethics approved the study in May 2021 (\#227624). In stages 3 and 4 , an informational letter and an informed consent form will be distributed to the participants to sign before the interview. The study results will be disseminated through publications, conference presentations, and national and local public forums to healthcare professionals, service managers, policymakers and refugee-supporting agencies.

\section{Strengths and limitations of this study}

The use of indepth interviews in this study will provide torture survivors with an opportunity to express their experiences of healthcare.

- The interpretative phenomenological analysis as a methodology is significant in the context of refugee studies because it emphasises being open to human experiences and the unique features of individual experiences.

- Reporting experiences of receiving surgical care and recounting experiences of torture may be difficult for participants, but this can be compensated by the fact that the patients will receive attention and have the opportunity to express themselves and convey their opinions.

- The use of a focus group approach to access healthcare professionals' experiences of providing care to torture survivors will provide the advantage of using group processes to explore and clarify individual opinions, which would be more difficult to do using one-to-one interviews.

- One limitation of this study may be the fact that the sample may represent only a subgroup of refugees who demonstrate willingness to participate in research, since severely traumatised patients such as torture survivors show reluctance to participate due to difficulties and resistance to talking about traumatic experiences.

\section{INTRODUCTION}

Millions of people are violently displaced from their homes due to wars and conflicts, persecution, and serious human rights violations, ${ }^{12}$ and a significant number of them have experienced torture. ${ }^{134}$ It is estimated that up to $35 \%$ of refugees and asylum seekers have possibly experienced torture. ${ }^{56}$

The United Nations ${ }^{7}$ defines torture as follows: 
Any act by which severe pain or suffering, whether physical or mental, is intentionally inflicted on a person for such purposes as obtaining from him or a third person information or a confession, punishing him for an act he or a third person has committed or is suspected of having committed, or intimidating or coercing him or a third person, or for any reason based on discrimination of any kind.

War trauma and torture are some of the most traumatic life experiences, and victims of torture belong to one of the most vulnerable groups of refugees. ${ }^{8}$ They are at an increased risk of developing long-term mental and physical health problems and post-traumatic stress disorder. ${ }^{59}$ Due to fear, humiliation, shame, lack of support and mistrust, many survivors do not disclose their stories of torture to healthcare providers. ${ }^{81011}$ The possibility of torture survival among refugees and asylum seekers in a healthcare context is quite high $^{412}$; however, healthcare professionals, especially those working in somatic departments as surgical units, are neither aware of nor trained to recognise the sequela of torture. ${ }^{1012} 13$ The identification of torture survivors is the first step in providing adequate health assistance to this group of patients. However, this process appears to depend on self-identification because healthcare professionals lack the competence needed for such identification. ${ }^{6} 121415$ This may reinforce distrust ${ }^{16}$ and avoidance of disclosure. ${ }^{11} 17$ When torture experiences remain undisclosed, a part of the healthcare needs of torture survivors remain unaddressed, which leads to inferior healthcare outcomes. ${ }^{18}$ Torture survivors have complex health needs due to a combination of physical and psychological symptoms they present, as well as aspects of comorbidity ${ }^{19}$ and somatisation, ${ }^{20}$ which may require additional medical expertise. ${ }^{21}$ Common physical injuries from torture, such as head injuries, fractures, contractures and ear injuries, and urination and defecation problems resulting from beatings, forced positions, electric shocks, burns, sexual torture and other torture methods often require surgical treatment. ${ }^{1022} 23$ Torture survivors undergoing treatment in departments that treat somatic diseases and include surgical units describe receiving varying diagnoses and experiencing numerous unsuccessful treatments that leave them confused and unsatisfied. ${ }^{1415182425}$ A lack of awareness and knowledge regarding torture survivors' complex needs ${ }^{2425}$ can result in a failure to understand and treat them and eventually to retraumatisation. ${ }^{26}{ }^{27}$ The events or interactions that occur during treatment can remind survivors of their previous traumatic experiences ${ }^{28}$ and can cause retraumatisation. Understanding the mechanism of retraumatisation is part of understanding the specific nature of the traumatic memory, which includes explicit memories of the torture (which one can retrieve consciously) and implicit memories of torture (which are non-conscious). ${ }^{29}$ Reactivation of trauma symptoms can take place due to a new traumatic experience or trauma-associated stimuli occurring during treatment. Trauma-associated stimuli during treatment may involve aspects of treatment such as interactions, environment, equipment used and pain related to medical procedures. ${ }^{29}$ Retraumatisation of torture survivors during surgical care has been addressed in the literature. ${ }^{27}{ }^{30}$ However, there is a lack of systematic knowledge synthesis and studies focusing on survivors' self-reported experiences, whereas we need clinical guidelines for preventing retraumatisation of torture survivors during treatment. In the extant literature, there are guidelines for psychiatric care for torture survivors, ${ }^{31}$ documentation of torture, ${ }^{32}$ treatment of pain from torture, ${ }^{33}$ and documentation of torture and cruel, inhuman or degrading treatment in children. ${ }^{34}$ However, guidelines that focus on the prevention of retraumatisation of torture survivors during surgical treatment are yet to established. For this purpose, systematisation of knowledge and development of guidelines are crucial. Such guidelines can improve decision-making skills, simplify medical decision-making and improve care by identifying current practices that maximise benefits and minimise harm for patients (eg, retraumatisation). ${ }^{35-38}$

While the amount of research on forced migrants has increased, the number of studies addressing the issues of torture survivors and retraumatisation remains limited, especially in somatic healthcare. ${ }^{10} 1239$ Understudied groups of refugees, such as torture survivors, may be particularly vulnerable, and it may be more urgent to give them attention in associated research. ${ }^{40}$ Previous studies that have incorporated participants' voices have yielded a greater understanding of the challenges faced by ethnic minority participants regarding their health and engagement in research. ${ }^{40}$

\section{Aim of the project}

The overall aim of this study is to develop and evaluate a set of guidelines for prevention of retraumatisation of torture survivors during surgical care. Using a four-stage qualitative-method design, we aim to (1) summarise previous studies on the experiences of torture survivors receiving treatment for somatic diseases to identify the triggers/reminders in the somatic healthcare context that can lead to retraumatisation; (2) identify clinical guidelines and recommendations to establish best practices and enhance the effective and safe treatment of torture survivors in somatic healthcare; (3) explore how survivors of torture experiences receive surgical healthcare in different contexts, such as in operating theatres, intensive care departments and outpatient clinics in their host countries; and (4) develop and assess the feasibility and acceptability of a set of clinical guidelines for prevention of retraumatisation of torture survivors in surgical departments.

In this project, we move from a general approach, which involves summarising torture survivors' experiences related to treatment in departments that treat physical diseases (stage 1) and outlining the existing guidelines and recommendations for treatment of torture survivors (stage 2), to a more specific approach, which 


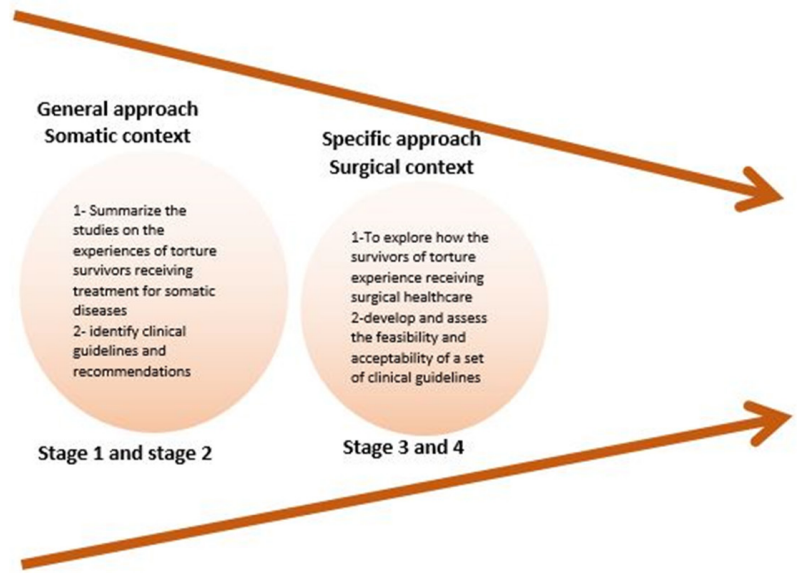

Figure 1 Diagram of the approach of the study.

involves examining torture survivors' experiences related to surgical treatment (stage 3 ). The project approach is outlined in figure 1.

In stage 3, we will explore torture survivors' experiences of receiving surgical healthcare and explore how eventual trauma-associated stimuli can trigger reactions associated with retraumatisation during treatment.

Stage 4 comprises two substages: (1) development of guidelines for prevention of retraumatisation of torture survivors under surgical treatment based on the findings from stages 1, 2 and 3; and (2) assessment of the feasibility and acceptability of the developed set of clinical guidelines based on the findings from focus group interviews with healthcare professionals working in surgical departments.

\section{METHODS AND ANALYSIS}

This project includes two systematic reviews that summarise the literature related to the experiences of torture survivors undergoing somatic treatment (stage 1) and the existing guidelines and recommendations regarding the treatment of torture survivors in somatic healthcare (stage 2). Stage 3 endeavours to explore the experiences of survivors of torture receiving surgical care through indepth interviews. Building on the earlier stages, in stage 4, we will develop and assess the feasibility and acceptability of a set of clinical guidelines that can help prevent retraumatisation of torture survivors in surgical departments through interdisciplinary focus group interviews.

The methods chosen for data collection and analysis will ensure the development of relevant guidelines for use in clinical practice. Stage 1 of the study has been

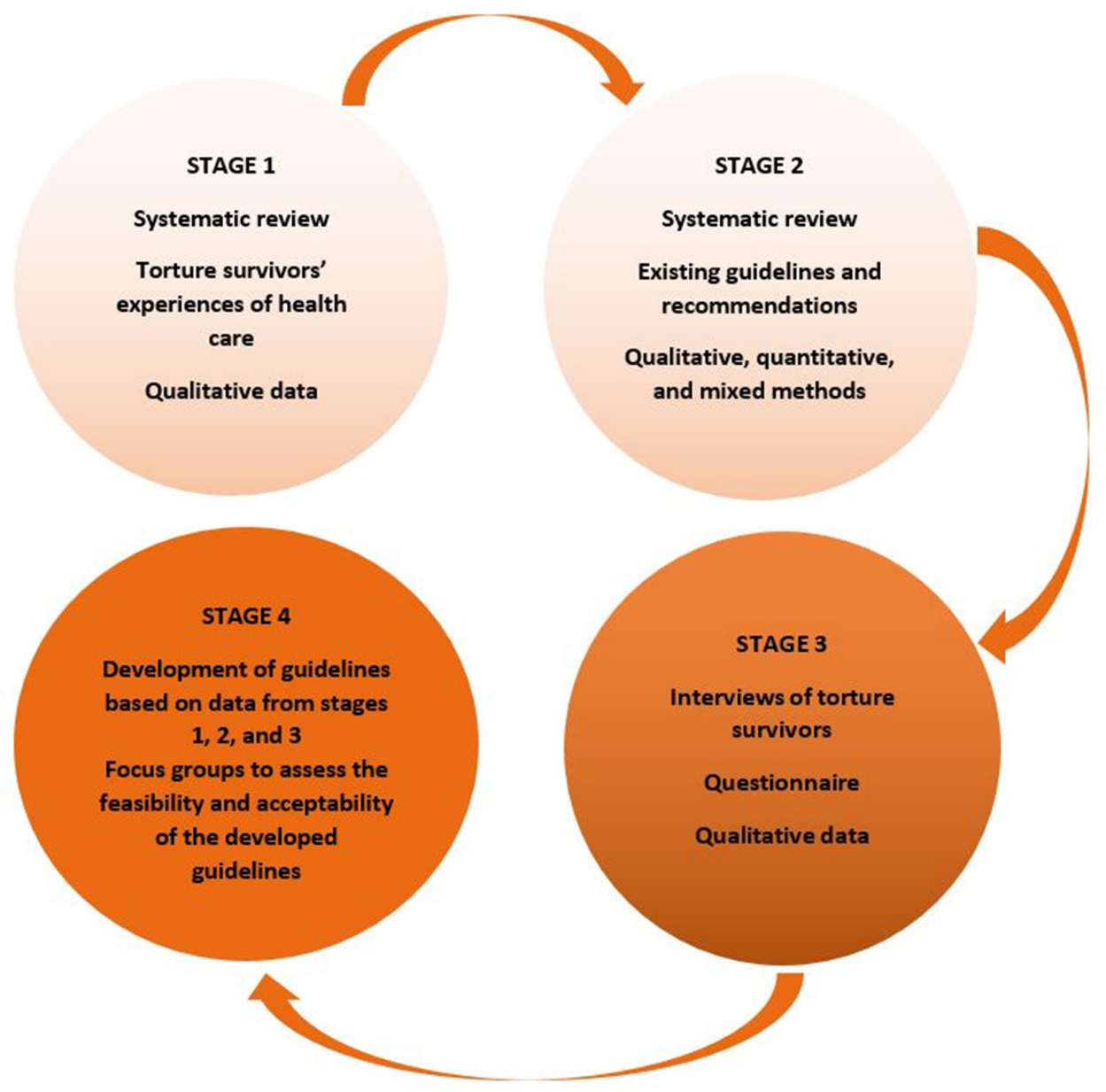

Figure 2 Diagram of the study design and methods employed in this project. 
completed and stage 2 is ongoing. The study is expected to be completed by December 2025. The flow of the study is presented in figure 2, and the PICO ( $\mathrm{P}$ (pacient, population or problem) I(Intervention) $\mathrm{C}$ (comparison) $\mathrm{O}$ (outcomes) ) used for stages 1, 2, 3 and 4 is presented in online supplemental table 1.

\section{Stage 1: uncovering the retraumatisation experiences of} torture survivors in somatic health: a systematic review

Stage 1 has been completed and published. ${ }^{41}$ The main objective of this stage was to summarise previous qualitative studies on retraumatising experiences of torture survivors undergoing treatment in somatic healthcare departments and uncover the triggers/reminders that can cause retraumatisation in the somatic healthcare context. Nine electronic bibliographical databases, including grey literature sources, were systematically searched in August 2019, which resulted in a total of 6326 studies. Of these, eight were included in the final review. A full description of the search strategy is provided in online supplemental table 2, and the search conducted during this stage is provided in online supplemental table 3 .

Dallam's healthcare retraumatisation model ${ }^{28}$ was used as a framework for data extraction and analysis, and $a$ retraumatisation model of torture survivors in healthcare was created. ${ }^{41}$ The study's findings indicated that torture survivors do not receive adequate healthcare and may experience challenges during treatment that can result in retraumatisation.

\section{Stage 2: clinical guidelines, suggestions and recommendations for treatment of torture survivors in somatic healthcare: a systematic review}

The objectives of this stage are as follows: (1) to summarise the existing guidelines and primary research studies that include recommendations and suggestions for treatment of torture survivors in somatic healthcare; and (2) to prepare a preliminary set of guidelines and recommendations for prevention of retraumatisation of torture survivors in somatic healthcare. This stage is currently in progress, and we are working to identify clinical guidelines and recommendations that can help clarify the best practices to be implemented and enhance our capacity to provide effective and safe treatment to torture survivors in somatic healthcare. The review protocol has been registered with the International Prospective Register of Systematic Reviews (PROSPERO) database (PROSPERO registration number CRD42020182783) and is being reported in compliance with the reporting guidance provided in the Preferred Reporting Items for Systematic Review and Meta-Analysis Protocols (PRISMA-P) statement. $^{42}$

\section{Study design}

This review will include studies that use qualitative, quantitative and mixed methods for data collection and data analysis. The review will summarise the clinical practice guidelines (CPGs) published since 2000 that address the treatment of torture survivors in somatic healthcare, using Medline, Cumulative Index to Nursing and Allied Health (CINAHL), Embase, PsycINFO, Web of Science, OpenSIGLE, WHO (International Clinical Trials Registry Platform), PTSDpubs, OpenGrey, Guidelines International Network and UpToDate. All grey literature ${ }^{43}$ will be covered by searching through publications for relevant associations and health organisations that have focused on torture survivors, such as the International Rehabilitation Council for Torture Victims (https://irct.org/), the Rehabilitation and Research Centre for Torture Victims (https://irct.org/), and the Center for Victims of Torture (https://www.cvt.org/).

A full description of the search strategy used is provided in online supplemental table 4 , and the search conducted during this stage is presented in online supplemental table 5 .

\section{Eligibility criteria}

Articles that report CPGs for treatment of torture survivors in somatic healthcare will be included in this study. Independent of study design, we also intend to include studies that involve refugees, asylum seekers and war survivors who have experienced psychological and/or physical torture, and provide recommendations for treatment of torture survivors in somatic healthcare in the discussion and conclusion chapters.

\section{Study setting}

The study settings included in this project will be all healthcare facilities within somatic care (ie, hospitals, healthcare centres, emergency departments, operating theatres, intensive care departments, surgical departments, general polyclinics and surgical polyclinics). CPGs designed only for psychiatric treatment will be excluded.

\section{Procedures}

Search results will be uploaded to Covidence, ${ }^{44}$ a webbased software program that assists researchers with screening and data extraction process. After removal of duplicates, eligible studies will be identified through title and abstract screening, which will be performed by five review authors (ACS, AKB, EKG, SOD and BS-N). Next, potentially relevant studies will be screened against the defined eligibility criteria based on full-text records by two review authors (ACS and AKB). Both the title/ abstract and full-text screening will be performed blindly by review, and disagreements will be resolved via discussion until a consensus is reached. If needed, a third reviewer will be consulted to achieve consensus (EKG). The PRISMA flow chart will be used to illustrate the flow of the study selection process. ${ }^{42}$

Data will be extracted from the selected studies independently by two reviewers (ACS and AKB) using a standard form. Any disagreements will be settled through discussion with a third reviewer (EKG). Extracted data will include specific aspects of the research question and cover the study details (title, author, publication details, 
location and language), study design, participant demographics, intervention characteristics, outcomes and the study's conclusions.

Data derived from the findings will be organised according to various themes and keywords based on the recommendations for treatment of torture survivors in somatic healthcare.

\section{Quality assessment}

\section{Assessment of risk of bias in the included studies}

Two authors (ACS and AKB) will independently assess the risk of bias in each primary research study using the critical appraisal checklist published by the Joanna Briggs Institute based on the research design used in each study. ${ }^{45-47}$ Any disagreements will be resolved by referral to a third review author (EKG). A 'risk of bias' table will be created for each included study.

\section{Quality assessment of included CPGs}

The quality of each CPG will be assessed using the Appraisal of Guidelines Research and Evaluation Version II (AGREE II) instrument. This instrument was developed to address variability issues in the quality of guidelines. It is a generic instrument used to assess the methodological rigour and transparency of the guideline development process. It is a widely accepted tool for evaluation of the rigour of the development and transparency of $\mathrm{CPGs}^{48-51}$ and includes 23 items organised into 6 domains, namely 'scope and purpose', 'stakeholder involvement', 'rigor of development', 'clarity of presentation', 'applicability' and 'editorial independence'. ${ }^{49}$ The purpose of AGREE II is to provide a framework that can be used to assess the quality of guidelines, provide a methodological strategy for the development of guidelines, and determine what information should be reported in the guidelines and how this should be carried out. ${ }^{52}$

To ensure a standardised approach, the reviewers will complete the AGREE II online training tutorials ${ }^{51} 53$ before performing quality assessment. A summary of the extracted data and AGREE scores will be presented in a descriptive table.

\section{Assessment of the overall confidence in the review findings}

Quality assessment will include an overall evaluation of the confidence in the findings in accordance with the GRADE-CERQual (Confidence in the Evidence from Reviews of Qualitative Research) approach (confidence in the evidence from reviews of qualitative research).$^{545}$ The CERQual assessment will consider the following study characteristics: (1) the methodological limitations of the studies; (2) the relevance of the included studies to the review question; (3) the coherence of the findings of the included studies; and (4) the adequacy of the data based on the richness and quantity of the data that support the findings. After completing an overall assessment of the confidence in the findings, we will proceed from the analyses conducted for each CERQual component to a final assessment. ${ }^{55}$ The findings will then be defined as coherent if they are found to be valid across studies with similar populations and contexts. Data will be defined as 'rich' if they provide details that improve our understanding of the treatment of torture survivors in somatic departments. In contrast, we will define data as 'thin' if they do not provide any details that improve our understanding. The assessment based on the evaluations of each of the four CERQual components will be summarised in four levels: high, moderate, low and very low. The CERQual approach will be employed by the review team to identify any familiarity with the evidence to draw reasonable conclusions regarding each CERQual component. This will benefit the synthesis of qualitative evidence and allow for reflection on the way in which the synthesis is conducted and the review findings are formulated..$^{55}$

\section{Data synthesis}

A summary of the evidence will be processed for each included study and the included guidelines. The recommendations identified across the guidelines and studies will be grouped into tables. The grouped interventions will be listed in descending order based on the number of guidelines and articles that have recommended them, and we will then determine areas of agreement. The evidence tables will provide an overall description of the studies, including their context, participants, outcomes and conclusions. A narrative synthesis ${ }^{56}$ will be used, which involves compiling the findings of all the included studies in a text narrative and the descriptions of the characteristics of the studies, including their context and validity. ${ }^{57}$ To ensure trustworthiness of the review's conclusions, we will use the published guidance for conducting narrative synthesis that was funded by the UK's Economic and Social Research Council. ${ }^{56}$

\section{Output (stage 2)}

The output will be a peer-reviewed article corresponding to the objectives, which will compound a part of the background for the clinical guideline development in stage 4 .

\section{Stage 3: the lived experiences of torture and experiences during surgical treatment}

The aim of this stage is to explore how survivors of torture experiences receive surgical healthcare in various contexts, such as in operating theatres, intensive care departments and outpatient clinics, and identify the triggers and protective factors involved in the process of retraumatisation.

\section{Study design}

We will conduct indepth interviews with a strategic sample of 8-12 torture survivors who have received surgical treatment. The choice of the sample size is based on the recommendations found in the literature for phenomenological studies that aim to obtain insights using comprehensive and 'rich' data. ${ }^{58}$ Although the sample recommended in an interpretative phenomenological analysis (IPA) study is of six to eight participants, ${ }^{60}{ }^{61}$ we 
will also take into account the richness of the individual cases to determine whether there is a need to increase the sample size. ${ }^{61}$

\section{Recruitment}

Following a multi-institutional recruitment in Norway, participants will be recruited from asylum institutions, health centres for refugees, district psychiatric centre and surgical departments of university hospitals. We will also contact the Health Centre for Undocumented Migrants, Red Cross Office, Church's Foundation (Stiftelsen Kirkens Bymisjon) and Migrations Health Centre for recruitment. Information about the project will be sent to various organisations to request their practical advice and support regarding recruitment. Aspects such as language, need for research assistants, provision of financial incentives and information to potential participants, and how to increase patients' willingness to participate will be discussed. Potential participants will be contacted by the healthcare professionals or social workers affiliated with their institution.

We will also recruit patients from the Central Operation and Day Surgery Center at Akershus University Hospital (Ahus). Torture survivors who have undergone surgical treatment in this institution will be contacted by a health secretary employed at Ahus. This will be carried out in consultation with the leading surgeon. Patients will receive written and oral information about the study and will have 2 weeks to reach out to the secretary with their decision. No reminders will be sent if there is no response.

We will contact the patients only after they have confirmed their willingness to participate in the study. Thereafter, we will have a conversation with them to inform them about the project and discuss any concerns they may have. In accordance with the recruitment strategies recommended by Gabriel et $a l^{40}{ }^{40}$ we will avoid including patients who have only recently arrived in Norway, as this could cause them to experience confusion and fear and overwhelm them. Further, the voluntary nature of participation will be explicitly stated and explained to the participants. We will ensure that they understand that their opportunity for treatment will not be impacted by their refusal to participate.

\section{Eligibility criteria}

The sample of this study comprises adult participants of various ages and both sexes who have been identified as survivors of torture or ill treatment according to the United Nation's 1984 definition, ${ }^{62}$ have been living in Norway for more than 3 months and have undergone surgical treatment. We will not include more than two participants from the same country to ensure data diversity. According to Pabilonia et $a l,{ }^{63}$ men and women are equally likely to experience torture, whereas, according to Higson-Smith and Bro, ${ }^{64}$ men experience higher levels of traumatic events and are more likely to have experienced torture. On the other hand, women are particularly at risk of organised and gender-based violence, including rape, and have been found to be more likely to have a higher number of negative reactions, ineffective coping mechanisms and difficulties in adapting after torture. ${ }^{63}$ Due to these differences with regard to sex and gender, it is crucial that our study include both men and women. Although certain studies report that most torture survivors are in their $30 \mathrm{~s},{ }^{63}$ we will include participants of different ages, as a history trauma can heighten older survivors' sensitivity to many aspects of the social and physical environments of healthcare. ${ }^{65}$ There is a lack of research on the experiences of ageing refugees even though they appear to be a vulnerable at-risk group. ${ }^{66}$ Studies have reported exacerbation of post-traumatic stress symptoms, even after several years of the traumatic events, instead of relief of symptoms. ${ }^{66}$ Older survivors of torture may have a different capacity for functioning and coping under treatment, which should be considered when designing treatment plans and managing the needs of such patients. ${ }^{65}$ These are important factors and will be addressed in this study.

\section{Data analysis}

The interviews will be audio-recorded and transcribed verbatim. The transcribed data will be managed/imported into NVivo, and the interviews will be analysed using IPA, which was developed by Smith et al. ${ }^{67}$ IPA is a rigorous methodology based on phenomenological principles and characterised by three qualities: it is idiographic, inductive and interrogative. ${ }^{6768}$ The methodology has salience with regard to studies on refugees as it emphasises being open to human experience. Using a phenomenological framework, the researchers aim to understand the experiences of people who have experienced torture. The IPA procedures will help the researcher become well acquainted with the data by developing codes and themes from them. The procedures will also allow the researcher to focus on the characteristics of each participant.

In the analysis, we will compare the findings derived from the interviews using the theoretical framework developed in stage 1 . As we expect that participant recruitment will take time, we will carry out our data analysis concurrently with the recruitment and interviewing processes.

\section{Output (stage 3)}

The output of this stage will be one peer-reviewed article corresponding to the objectives, which will inform the development of clinical guidelines.

\section{Stage 4: developing clinical guidelines for preventing retraumatisation during surgical treatment of torture survivors}

The aims of stage 4 are to (1) develop and (2) assess the feasibility and acceptability of a set of clinical guidelines and thereby prevent retraumatisation of torture survivors in surgical departments. In stage 4a, we will develop a set of clinical guidelines for prevention of retraumatisation of such patients based on our findings from stages 1, 2 and 3. As the identification of torture survivors is 


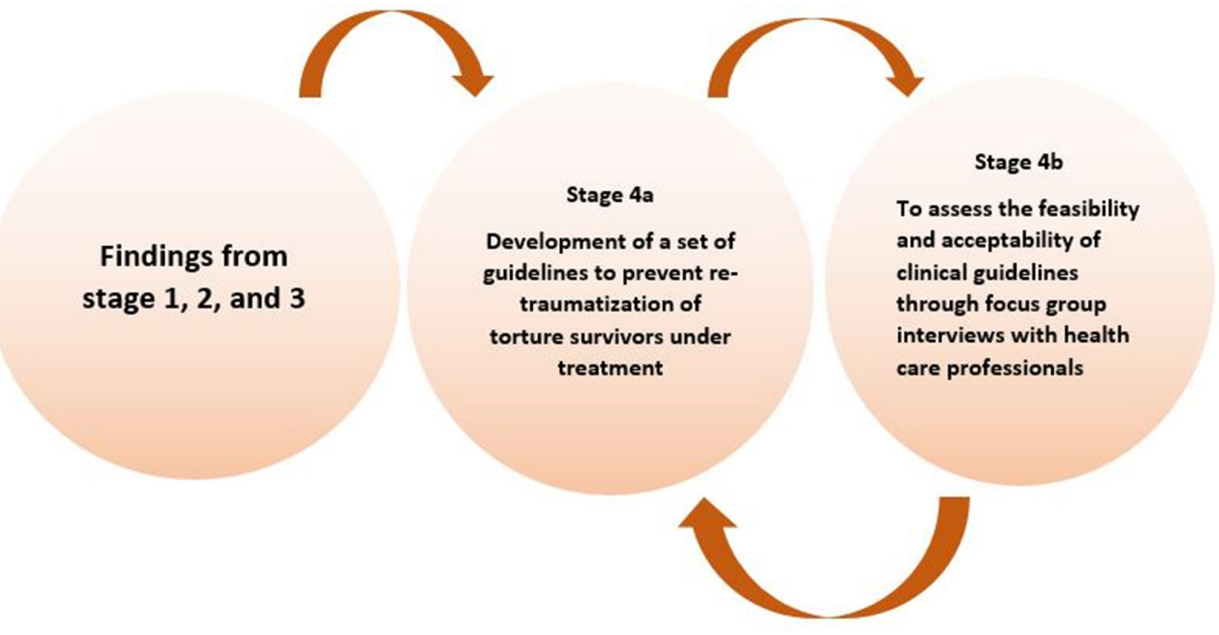

Figure 3 Process for the development of clinical guidelines.

central to preventing retraumatisation, we will prepare a checklist that will help healthcare professionals with this process. The checklist will also be based on our findings from stages 1, 2 and 3, and it will be presented as a supplement to the guidelines. In stage $4 \mathrm{~b}$, we will explore the challenges and dilemmas that healthcare professionals working in surgical departments face during the surgical treatment of torture survivors. Accordingly, we will identify the necessary content of clinical guidelines for prevention of retraumatisation of such patients. Based on the findings from stage $4 \mathrm{a}$, we will consider modifying the structure and content of the guidelines to increase its practical usability. This process is illustrated in figure 3 .

\section{Methods}

The findings from stages 1,2 and 3 will be the basis for the development of a set of clinical guidelines for preventing retraumatisation of torture survivors during treatment in surgical departments. Furthermore, we will organise interdisciplinary semistructured focus group interviews with healthcare professionals to explore the challenges and dilemmas they face with respect to encounters between healthcare providers and torture survivors. The feasibility and acceptability of the proposed clinical guidelines will also be discussed with the healthcare providers.

In stage $4 \mathrm{a}$, we will follow the Institute of Medicine's proposed standards for trustworthy guidelines. ${ }^{69}$ According to these guidelines, a set of guidelines should:

- Be based on findings derived from a systematic review of the existing evidence.

- Be developed by a multidisciplinary group of experts and representatives from the affected groups (in this case, torture survivors).

- Consider patient preferences.

- Be based on an explicit and transparent process to minimise biases and conflicts of interest.

- Provide a good explanation of the relationships between different care options and health outcomes.

- Provide ratings of both the quality of evidence and the strength of recommendations.
- Be reconsidered and revised as appropriate when important new evidence appears. ${ }^{69} 70$

We will follow these principles when developing the guidelines and we will also use the standards and guidance for developing trustworthy CPGs available on the MAGICapp platform (www.magicproject.org) to ensure trustworthiness. This guide will help us choose appropriate presentation formats, ensure efficient dissemination to healthcare professionals at the point of care and prevent the guidelines from becoming outdated quickly. ${ }^{69}$ To address the limitations in the development of guidelines, we will use the research programme MAGIC (Making GRADE the Irresistible Choice), which includes a conceptual framework and tools that can be used to facilitate the creation, dissemination and dynamic updating of trustworthy guidelines. Modifications to the guidelines, such as recommendation updates, will lead to automatic alterations with minimal additional work for the authors and publishers, greatly facilitating their dynamic updating.

\section{Recruitment}

In stage $4 \mathrm{~b}$, we will organise interdisciplinary focus group interviews with healthcare providers from surgical departments, including nurses, physiotherapists, surgeons and anaesthesiologists representing operating theatres, postsurgical departments, surgical intensive care and surgical outpatient clinics from various hospitals in Health SouthEast (HSØ). About $70 \%$ of all immigrants living in Norway live in South-East's recording area, and hospitals that have an agreement with HS $\varnothing$ have a high probability of treating torture survivors. ${ }^{71}$

Participants will be recruited by contacting the surgical services of three hospitals. We will arrange three focus groups, which will be conducted with five participants per group. This number is recommended by contemporary focus group research; smaller groups are easier to manage when topics are highly sensitive and are more likely to encourage participation by all members. ${ }^{72}$ Informed consent will be obtained before each focus group. 
One of the authors (ACS) will act as the moderator and two others (AKB and TD-M) will take field notes. The sessions will be scheduled as per participants' convenience and held at various localities, specifically at Ahus, Oslo Metropolitan University and elsewhere, depending on the preference of the participants.

\section{Data analysis}

In stage $4 \mathrm{~b}$, data analyses will be conducted by four authors (ACS, TD-M, EKG and AKB). The entire research team will participate in the interpretation and discussion of the results.

Interviews will be audio-recorded and transcribed verbatim. The transcribed data will then be imported into and managed using NVivo, and text condensation analyses will be performed. ${ }^{74-76}$ This approach involves defining units, subcategories, themes, and the manifest and latent content of the text. The raw data will be revisited regularly to ensure that the resulting categories are based on the data. ${ }^{77}$ The method represents a pragmatic approach and is inspired by phenomenological ideas. The procedure consists of several steps: (1) developing themes from the total impression from the data; (2) developing codes from the themes by identifying the meaning of the themes; (3) condensation (from code to meaning); and (4) synthesising (from condensation to descriptions and concepts). Systematic text condensation is a strategy used for analysis that has been developed from the common principles of most of the methods used for analysis of qualitative data. ${ }^{78}$ Compared with other frequently employed qualitative methods, such as a thematic analysis or the theoretical methodological framework, this method offers the researcher a process characterised by intersubjectivity, reflexivity and feasibility while maintaining a high level of methodological rigour. ${ }^{78}$ The process of text condensation is illustrated in figure 4.

Based on the findings from stage $4 \mathrm{~b}$, we will consider whether it is necessary to revise the developed guidelines.

\section{Output (stage 4)}

This stage will conclude with the formulation of a set of guidelines based on our findings (from the focus groups and stages 1, 2 and 3) for prevention of retraumatisation of torture survivors during surgical care. We expect that the focus group findings will provide important data about the challenges and dilemmas that healthcare professionals face during surgical treatment of torture survivors. We will also obtain information about the practical value of the developed guidelines.

\section{PATIENT AND PUBLIC INVOLVEMENT}

The design of this study protocol considers the suggestions of an important and recent report from the Norwegian Red Cross which demonstrates the importance of the topic and the significance of researching the topic. ${ }^{79}$ This project has also been discussed by the leaders of professional groups for surgical nurses, intensive care nurses and anaesthesia nurses who belong to the Norwegian Nurses Association. By recruiting participants through organisations such as the Health Centre for Undocumented Migrants, Red Cross Office, Church's Foundation (Stiftelsen Kirkens Bymisjon) and Migrations Health Centre, all of which work with torture survivors, we will involve public statements in our analysis. The review in stage 1 amplifies the voices of survivors using previous studies, and the interviews with the participants in stage 3 will provide a form of patient statement.

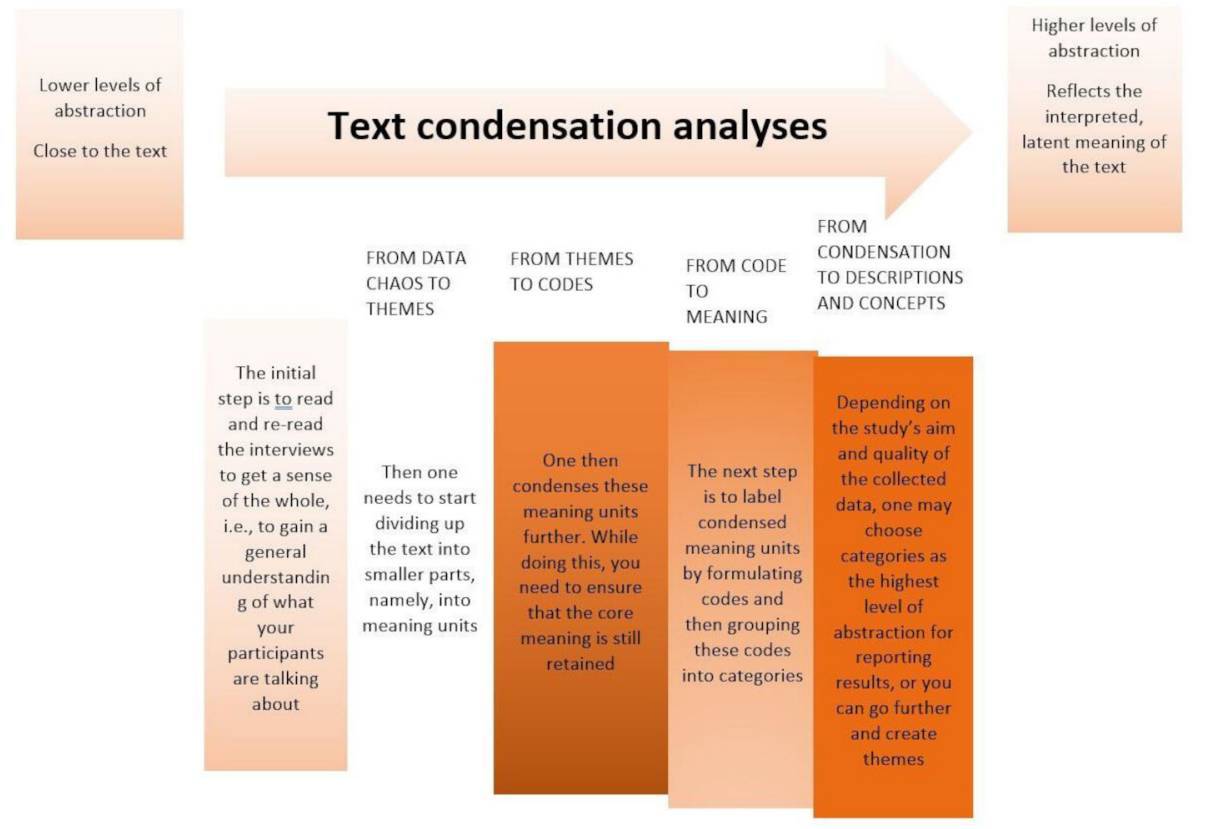

Figure 4 Text condensation analysis. 


\section{ETHICS AND DISSEMINATION}

This project complies with the principles stated in the Declaration of Helsinki. ${ }^{80}$ We applied to the Regional (South-East C) Committee for Medical and Health Research Ethics (REC), the Data Protection Official for Research (Norsk Senter for Forskningsdata (NSD)) and the hospital data protection officer (Personvernombud (PVO)) for permission to carry out this study. Ethics approvals were obtained from the REC in May 2021 (\#227624) and from the PVO in June 2021.

Following ethical norms is particularly important in this study because research with refugees involves conceptual, ethical and methodological issues. ${ }^{408182}$ These include the ethical considerations in the development of participatory research designs; the relationship between research, practice and policy ${ }^{83}$; and the issues around informed consent with regard to refugees' capacity for autonomy and the notion of reciprocation in refugee research. ${ }^{84}$ The confidentiality of the interviews will be specified to both the participants and the interpreters. It will be emphasised that participation is voluntary, and the participants will be required to sign a consent form. When presenting the results, anonymity of the participants will be maintained through the use of pseudonyms, and they will be described in a way that ensures they cannot be identified. The recorded interviews and transcripts will be stored in accordance with the regulations of the Norwegian Centre for Research Data. ${ }^{85}$

The vulnerability of people who have survived torture will be taken into consideration, specifically in terms of the researcher's responsibility to avoid strain ('not harm') and to protect the participants from distress. ${ }^{84}$ Reporting their experiences of receiving surgical care and remembering experiences of torture can be emotionally challenging, but the fact that the patients will receive attention and have the opportunity to express themselves can compensate for this. We will make efforts to ensure that the participants fully understand the implications of being involved in this study. The informed consent procedure can discourage participants from participating in the project because signing consent forms may jeopardise their anonymity. This may cause challenges for recruitment. Consent forms can be difficult to translate into multiple languages in a comprehensible manner, particularly if patients have low levels of literacy. We will use professional translators for this. With respect to gender, certain female refugee subgroups may be shy, afraid or ashamed and this will be addressed by employing a female researcher to perform the interviews and a female interpreter if needed.

Although experiences of retraumatisation among torture survivors often reflect the dynamics of the clinical setting rather than the research context, ${ }^{86}$ we will make special considerations regarding the vulnerability of this group of participants and arrange for them to contact a healthcare professional in case they have a negative reaction as a consequence of the interview. We will ensure that the participants understand that the research will not influence their housing, resettlement, relocation or status determination procedures. To protect participants' safety and prevent stigmatisation, social exclusion or racism, we will be careful about the kind of information we collect. We will not collect names, addresses or specific locations and exact dates unless necessary.

The study results will be disseminated through publications, conference presentations, and national and local public forums to healthcare professionals, service managers, policymakers and refugee-supporting agencies.

Contributors ACS and AKB conceptualised the study and wrote the study protocol. ACS and AKB designed the details of the study with substantial contributions from EKG. ACS, EKG, TD-M, JS and AKB are investigators responsible for the project. ACS and AKB prepared the first draft of the manuscript. ACS, EKG, TD-M, JS, BS-N, SOD, $M A$ and $A K B$ contributed to critically revising the manuscript regarding important intellectual content, approved the final version to be published and agree to be accountable for the work as guarantors. The corresponding author attests that all listed authors meet the authorship criteria and no others who meet the criteria have been omitted.

Funding This work is funded by a PhD fellowship from the Institute of Nursing and Health Promotion, Oslo Metropolitan University, Norway, and Akershus University Hospital (Ahus), Norway. The Norwegian Nursing Association (NSF) will be funding stages 3 and 4 .

Competing interests None declared.

Patient consent for publication Not required.

Provenance and peer review Not commissioned; externally peer reviewed.

Supplemental material This content has been supplied by the author(s). It has not been vetted by BMJ Publishing Group Limited (BMJ) and may not have been peer-reviewed. Any opinions or recommendations discussed are solely those of the author(s) and are not endorsed by BMJ. BMJ disclaims all liability and responsibility arising from any reliance placed on the content. Where the content includes any translated material, BMJ does not warrant the accuracy and reliability of the translations (including but not limited to local regulations, clinical guidelines, terminology, drug names and drug dosages), and is not responsible for any error and/or omissions arising from translation and adaptation or otherwise.

Open access This is an open access article distributed in accordance with the Creative Commons Attribution Non Commercial (CC BY-NC 4.0) license, which permits others to distribute, remix, adapt, build upon this work non-commercially, and license their derivative works on different terms, provided the original work is properly cited, appropriate credit is given, any changes made indicated, and the use is non-commercial. See: http://creativecommons.org/licenses/by-nc/4.0/.

\section{ORCID iDs}

Ana Carla Schippert http://orcid.org/0000-0002-3993-2337

Bente Sparboe-Nilsen http://orcid.org/0000-0002-8983-812X

Ann Kristin Bjørnnes http://orcid.org/0000-0002-5356-3873

\section{REFERENCES}

1 Mollica RF. Surviving torture. N Engl J Med Overseas Ed 2004;351:5-7.

2 FN Sambandet. United Nations Association of Norway [Internet], 2021. Available: https://www.fn.no/tema/flyktninger

3 Masmas TN, Student E, Buhmann C. Asylum seekers in Denmark. Torture 2008;18:77-86.

4 Sanders J, Schuman MW, Marbella AM. The epidemiology of torture: a case series of 58 survivors of torture. Forensic Sci Int 2009;189:e1-7.

5 Sigvardsdotter E, Malm A, Tinghög P, et al. Refugee trauma measurement: a review of existing checklists. Public Health Rev 2016;37:10.

6 Luci M, Di Rado D. The special needs of victims of torture or serious violence: a qualitative research in Eu. J Immigr Refug Stud 2020;18:405-20.

7 Assembly UG. Convention against torture and other cruel, inhuman or degrading treatment or punishment. 13. United Nations: UN General Assembly, 1984. 
8 Dalgaard NT, Bjerre K, Thøgersen MH. Twenty seven years of treating survivors of torture and organized violence - associations between torture, gender and ethnic minority status among refugees referred for treatment of PTSD. Eur J Psychotraumatol 2021;12:1904712.

9 Steel Z, Chey T, Silove D, et al. Association of torture and other potentially traumatic events with mental health outcomes among populations exposed to mass conflict and displacement: a systematic review and meta-analysis. JAMA 2009;302:537-49.

10 Clarysse K, Grosber M, Ring J, et al. Skin lesions, differential diagnosis and practical approach to potential survivors of torture. $J$ Eur Acad Dermatol Venereol 2019;33:1232-40.

11 Shannon P, O'Dougherty M, Mehta E. Refugees' perspectives on barriers to communication about trauma histories in primary care. Ment Health Fam Med 2012;9:47.

12 Eisenman DP, Keller AS, Kim G. Survivors of torture in a general medical setting: how often have patients been tortured, and how often is it missed? West J Med 2000;172:301.

13 Amris K, Torp-Pedersen S, Rasmussen OV. Long-term consequences of falanga torture--what do we know and what do we need to know? Torture 2009;19:33-40.

14 Amris K, Jones LE, Williams ACdeC, ACdC W. Pain from torture: assessment and management. Pain Rep 2019;4:e794.

15 Piwowarczyk L, Moreno A, Grodin M. Health care of torture survivors. JAMA 2000;284:539-41.

16 Behnia B. Trust building from the perspective of survivors of war and torture. Soc Serv Rev 2004;78:26-40.

17 Shannon PJ. Refugees' advice to physicians: how to ask about mental health. Fam Pract 2014;31:462-6.

18 Board D, Childs S, Boulton R. Torture-survivors' experiences of healthcare services for pain: a qualitative study. $\mathrm{Br} J \mathrm{Pain}$ 2021;15:291-301.

$19 \mathrm{Im} \mathrm{H}$, Swan LE, Warsame AH, et al. Risk and protective factors for comorbidity of PTSD, depression, and anxiety among Somali refugees in Kenya. Int J Soc Psychiatry 2020:20764020978685.

20 Sambucini D, Aceto P, Begotaraj E, et al. Efficacy of psychological interventions on depression anxiety and somatization in migrants: a meta-analysis. J Immigr Minor Health 2020;22:1320-46.

21 Murray B, O'Donnell C. Nursing care in the acute hospital setting: survivors of torture. Advances in Mental Health 2013;11:188-96.

22 Punamäki R-L, Qouta SR, El Sarraj E. Nature of torture, PTSD, and somatic symptoms among political ex-prisoners. J Trauma Stress 2010;23:532-6.

23 Weisleder P, Rublee C. The neuropsychological consequences of armed conflicts and torture. Curr Neurol Neurosci Rep 2018;18:9.

24 Wilson JP, Drozdek B. The treatment of traumatized asylum seekers, refugees and war and torture victims. In: Broken spirits. Taylor \& Francis Ltd: Routledge, 2004

25 Juhler M. Surgical approach to victims of torture and PTSD. In Broken spirits. Routledge, 2004: 631-8.

26 Esala JJ, Vukovich MM, Hanbury A, et al. Collaborative care for refugees and torture survivors: key findings from the literature. Traumatology 2018;24:168.

27 Storvik AG. Torturtraumer på sykehuset. Dagens medisin 2007.

28 Dallam SJ. A model of the retraumatization process: a meta-synthesis of childhood sexual abuse survivors' experiences in healthcare. University of Kansas, 2010.

29 Schock K, Knaevelsrud C. Retraumatization: the vicious circle of intrusive memory. Hurting Memories and Beneficial Forgetting: Elsevier, 2013: 59-70.

30 Glad KA. Traumepåminnere. Tidsskrift for Norsk psykologforening 2017:54:648-52.

31 Kinzie JD. Guidelines for psychiatric care of torture survivors. Torture 2011;21:18-26.

32 Furtmayr H, Frewer A. Documentation of torture and the Istanbul protocol: applied medical ethics. Med Health Care Philos 2010;13:279-86.

33 Baird E, Williams ACdeC, Hearn L, et al. Interventions for treating persistent pain in survivors of torture. Cochrane Database Syst Rev 2017;8:CD012051.

34 den Otter JJ, Smit Y, dela Cruz LB, et al. Documentation of torture and cruel, inhuman or degrading treatment of children: a review of existing guidelines and tools. Forensic Sci Int 2013;224:27-32.

35 Laine C, Taichman DB, Mulrow C. Trustworthy clinical guidelines. Ann Intern Med 2011:154:774-5.

36 Elwyn G, Quinlan C, Mulley A. Trustworthy guidelines-excellent; customized care tools-even better. BMC Bedicine 2015;13:1-5

37 Ingemansson M, Bastholm-Rahmner P, Kiessling A. Practice guidelines in the context of primary care, learning and usability in the physicians' decision-making process - a qualitative study. BMC Fam Pract 2014:15:1-10.
38 Alonso-Coello P, Schünemann HJ, Moberg J, et al. Grade evidence to decision (ETD) frameworks: a systematic and transparent approach to making well informed healthcare choices. 1: introduction. BMJ;2016:i2016.

39 Jacobs $U$, lacopino $\mathrm{V}$. Torture and its consequences: a challenge to clinical neuropsychology. Prof Psychol 2001;32:458-64.

40 Gabriel P, Kaczorowski J, Berry N. Recruitment of refugees for health research: a qualitative study to add refugees' perspectives. Int $J$ Environ Res Public Health 2017;14:125.

41 Schippert ACSP, Grov EK, Bjørnnes AK. Uncovering retraumatization experiences of torture survivors in somatic health care: a qualitative systematic review. PLoS One 2021;16:e0246074.

42 Page MJ, McKenzie JE, Bossuyt PM, et al. Updating guidance for reporting systematic reviews: development of the PRISMA 2020 statement. J Clin Epidemiol 2021;134:103-12.

43 Paez A. Gray literature: an important resource in systematic reviews. J Evid Based Med 2017;10:233-40.

44 Covidence. Sign in to Covidence. Available: https://www.covidence. org/reviews/active

45 Critical appraisal tools [Internet]. The Joanna Briggs Institute. Adelaide, Australia, 1996.

46 WMd S, Secoli SR, VAdA P. The Joanna Briggs Institute approach for systematic reviews. Revista Latino-Americana de Enfermagem 2018;26.

47 Jordan Z, Lockwood C, Munn Z, et al. The updated Joanna Briggs Institute model of evidence-based healthcare. Int J Evid Based Healthc 2019;17:58-71.

48 Siering U, Eikermann M, Hausner E, et al. Appraisal tools for clinical practice guidelines: a systematic review. PLoS One 2013;8:e82915.

49 Brouwers MC, Kho ME, Browman GP, et al. Agree II: advancing Guideline development, reporting and evaluation in health care. CMAJ 2010;182:E839-42.

50 Brouwers MC, Kerkvliet K, Spithoff K, et al. The agree reporting checklist: a tool to improve reporting of clinical practice guidelines. BMJ 2016;352:11152.

51 Brouwers MC, Kho ME, Browman GP, et al. Development of the agree II, part 2: assessment of validity of items and tools to support application. CMAJ 2010;182:E472-8.

52 Consortium ANS. Appraisal of guidelines for research \& evaluation II AGREE II Instrument. The Agree Research Trust, 2009.

53 Makarski J, Brouwers MC. The agree enterprise: a decade of advancing clinical practice guidelines. Implementation Science 2014;9:1-3.

54 Lewin S, Glenton C, Munthe-Kaas H, et al. Using qualitative evidence in decision making for health and social interventions: an approach to assess confidence in findings from qualitative evidence syntheses (GRADE-CERQual). PLoS Med 2015;12:e1001895.

55 Lewin S, Bohren M, Rashidian A, et al. Applying GRADE-CERQual to qualitative evidence synthesis findings-paper 2: how to make an overall CERQual assessment of confidence and create a summary of qualitative findings table. Implement Sci 2018;13:10.

56 Popay J, Roberts H, Sowden A. Guidance on the conduct of narrative synthesis in systematic reviews: a product from the ESRC methods programme version 1. Computer Science 2006;1:b92.

57 Campbell M, Katikireddi SV, Sowden A, et al. Improving conduct and reporting of narrative synthesis of quantitative data (ICONS-Quant): protocol for a mixed methods study to develop a reporting guideline. BMJ Open 2018;8:e020064.

58 Hennink MM, Kaiser BN, Marconi VC. Code saturation versus meaning saturation: how many interviews are enough? Qual Health Res 2017;27:591-608.

59 Sim J, Saunders B, Waterfield J, et al. Can sample size in qualitative research be determined a priori? Int J Soc Res Methodol 2018;21:619-34.

60 Alase A. The interpretative phenomenological analysis (ipa): a guide to a good qualitative research approach. International Journal of Education and Literacy Studies 2017;5:9-19.

61 Pietkiewicz I, Smith JA. A practical guide to using interpretative phenomenological analysis in qualitative research psychology. Psychological Journal 2014:20:7-14.

62 Protocol I. Manual on the effective investigation and documentation of torture and other cruel, inhuman or degrading treatment or punishment. United Nations. 1999.

63 Pabilonia W, Combs SP, Cook PF. Knowledge and quality of life in female torture survivors. Torture 2010;20:4-22.

64 Higson-Smith C, Bro F. Tortured exiles on the streets: a research agenda and methodological challenge. Intervention 2010;8:14-28.

65 Teshuva K, Wells Y. Aged care managers' perceptions of staff preparedness for caring for older survivors of genocide and mass trauma in Australia: how prepared are aged care workers? Australas J Ageing 2017;36:E20-2. 
66 Dubus N. "I feel like her daughter not her mother": Ethnographic trans-cultural perspective of the experiences of aging for a group of Southeast Asian refugees in the United States. J Aging Stud 2010;24:204-11.

67 Smith JA, Jarman M, Osborn M. Doing interpretative phenomenological analysis. Qualitative Health Psychology: Theories and Methods, 1999: 218-40.

68 Quinn C, Clare L. Interpretive phenomenological analysis. In: Nursing research designs and methods, 2008: 375-84.

69 Vandvik PO, Brandt L, Alonso-Coello P, et al. Creating clinical practice guidelines we can trust, use, and share: a new era is imminent. Chest 2013;144:381-9.

70 Steinberg E, Greenfield S, Wolman DM. Clinical practice guidelines we can trust. National Academies Press;, 2011.

71 Helse-sørøst. LIKEVERDIGE HELSETJENESTER - Fokus på innvandrere/norskfødte Med innvandrerbakgrunn, 2009. Available: www.helse-sorost.no

72 Guest G, Namey E, Taylor J, et al. Comparing focus groups and individual interviews: findings from a randomized study. Int $J$ Soc Res Methodol 2017;20:693-708.

73 Morgan DL. Focus groups as qualitative research, 2013.

74 Graneheim UH, Lundman B. Qualitative content analysis in nursing research: concepts, procedures and measures to achieve trustworthiness. Nurse Educ Today 2004;24:105-12.

75 Graneheim UH, Lindgren B-M, Lundman B. Methodological challenges in qualitative content analysis: a discussion paper. Nurse Educ Today 2017;56:29-34.

76 Lindgren B-M, Lundman B, Graneheim UH. Abstraction and interpretation during the qualitative content analysis process. Int $\mathrm{J}$ Nurs Stud 2020;108:103632.
77 Kvale S, Brinkmann S. Interviews: learning the craft of qualitative research interviewing. Sage, 2009.

78 Malterud K. Systematic text condensation: a strategy for qualitative analysis. Scand J Public Health 2012;40:795-805.

79 Moa Nyamwathi Lønning AEAolL. Torturert OG glemt? Identifsering OG rehabilitering AV torturutsatte I Norge Røde Kors, 2020.

80 Goodyear MDE, Krleza-Jeric K, Lemmens T. The Declaration of Helsinki. . British Medical Journal Publishing Group, 2007: 335. 624-5.

81 Dickson-Swift V, James EL, Kippen S, et al. Risk to researchers in qualitative research on sensitive topics: issues and strategies. Qual Health Res 2008;18:133-44.

82 Dickson-Swift V, James EL, Kippen S, et al. Doing sensitive research: what challenges do qualitative researchers face? Qualitative Research 2007;7:327-53.

83 Gifford SM, Bakopanos C, Kaplan I, et al. Meaning or measurement? researching the social contexts of health and settlement among newly-arrived refugee youth in Melbourne, Australia. J Refug Stud 2007;20:414-40.

84 RAIO. Interviewing survivors of torture and other severe trauma, 2021. Available: https://www.uscis.gov/sites/default/files/document/ foia/Interviewing_-_Survivors_of_Torture_LP_RAIO.pdf: U.S. Citizenship and Immigration Services [Accessed 2021].

85 NSD. Norwegian Centre for Research Data [Internet]. Available: https://www.nsd.no/ [Accessed 20 May 2021].

86 Dehghan R, Wilson J. Healthcare professionals as gatekeepers in research involving refugee survivors of sexual torture: an examination of the ethical issues. Dev World Bioeth 2019;19:215-23. 